\title{
DYNAMIC LARGE-SIGNAL I-V ANALYSIS AND NON-LINEAR MODELLING OF ALGAN/GAN HEMTS
}

\author{
E. Chigaeva*, N. Wieser", W. Walthes ${ }^{*}$, M. Grözing ${ }^{*}$, and M. Berroth ${ }^{*}$ \\ H. Roll ${ }^{* *}$, O. Breitschädel ${ }^{* *}$, J. Off ${ }^{* *}$, B. Kuhn ${ }^{* *}$, F. Scholz ${ }^{* *}$, and H. Schweizer ${ }^{* *}$ \\ * Institute of Electrical and Optical Communication Engineering, University of Stuttgart, Pfaffenwaldring 47, \\ D-70569 Stuttgart, Germany, Tel.: +49 711685 7912, Fax: +49 7116857900 , \\ e-mail: nikolai.wieser@int.uni-stuttgart.de
}

*** Physics Department, $4^{\text {th }}$ Institute, University of Stuttgart, Pfaffenwaldring 57, D-70569 Stuttgart, Germany

\begin{abstract}
For AlGaN/GaN high electron mobility transistors (HEMTs), the voltage and current waveforms at $\mathrm{CW}$ large-signal operation at $5 \mathrm{GHz}$ have been reconstructed from experimental magnitude and phase information on fundamental and higher harmonics of transmitted and reflected signals. To compare with the $D C$ behaviour, the clipped waveforms have accurately been analysed to recover the dynamic output characteristics in view of dispersion effects related to self-heating. In conjunction with small-signal Sparameter data, the large-signal experimental results have been used in an attempt to apply a HEMT largesignal model, showing satisfactory agreement of simulated and measured characteristics at least in regions where self-heating is not much pronounced.
\end{abstract}

\section{INTRODUCTION}

It turned out that strong internal electric fields due to spontaneous and piezoelectric polarisations are responsible for the formation of the two-dimensional electron gas at the $\mathrm{AlGaN} / \mathrm{GaN}$ interface of respective HEMT structures, showing a sheet carrier concentration up to $2.0 \cdot 10^{13} \mathrm{~cm}^{-2}$ for $\mathrm{Al}$ mole fractions of $30 \%$ even without any additional doping (1). Although this and many other basic properties of nitride-based HEMTs are well understood by now, the origin of the so-called power compression or power slump, i.e. a lower RF-power than expected from DC-performance, is still an open question $(2,3)$. Dispersion may also arise from self-heating effects due to the sapphire substrate which has worse thermal conductivity but at lower cost than SiC. Comparatively less efforts have yet been done in the field of large-signal modelling in view of circuit design (4).

In this paper, we present results of both large-signal experimental analysis and modelling. A microwave transition analyser was used to measure the magnitude and phase of the fundamental and four higher harmonics of transmitted and reflected signals, yielding the RF time domain waveform information and thus the RF large-signal transfer and output characteristics $(5,6)$. Due to the above mentioned discrepancies between DC and RF operation, these dynamic experimental data appear to be more appropriate to be used for modelling purposes. For this, a first attempt to apply a HEMT large-signal model, which describes the current source and non-linear capacitances by analytical equations $(6,7)$, is presented finally. The transistor under investigation consisted of a $\mathrm{Al}(0.30) \mathrm{Ga}(0.70) \mathrm{N} / \mathrm{GaN}(30 \mathrm{~nm} / 2 \mu \mathrm{m})$ heterostructure with an additional $\mathrm{GaN}$ cap layer on top of the barrier, grown on sapphire substrate by metal organic vapour phase epitaxy. The gate length and width were 1 and $250 \mu \mathrm{m}$, respectively. Details on the metalization process may be found in (8).

\section{EXPERIMENTAL RESULTS}

Figure 1 shows the dynamic large signal transfer characteristics and respective waveforms (i.e., gate voltage, $\mathrm{V}_{\mathrm{gs}}$, and drain current, $\mathrm{I}_{\mathrm{ds}}$ ) measured at $5 \mathrm{GHz}$ and various input power levels. The time delay between the input and output signals has been corrected according to ref. (9). As in the case of GaAs MODFETs (6), the transfer characteristics does not significantly depend on the input power except the varying current swing. With increasing input power, i.e. with increasing contribution of higher harmonics due to compression, the time delay correction used becomes more and more inaccurate and thus the $\mathrm{I}_{\mathrm{ds}}-\mathrm{V}_{\mathrm{gs}}{ }^{-}$ trajectory becomes a loop. 
These measurements have been performed step by step at various drain voltages, $\mathrm{V}_{\mathrm{ds}}$, but constant $\mathrm{V}_{\mathrm{gs}}=-2.5 \mathrm{~V}$ and constant power levels, and the resulting data used to reconstruct the dynamic output characteristics to compare with the DC I-V's. Focussing on self-heating related dispersion, we restricted our measurements to an input voltage swing up to $\mathrm{V}_{\mathrm{gs}}=-0.5 \mathrm{~V}$ where the channel is not fully open and therefore possible open channel current compression at RF operation, see e.g. (2), should not be pronounced.

As shown in Figure 2, the current deduced from the power data is somewhat lower at low $\mathrm{V}_{\mathrm{ds}}$ due to selfbiasing (5) at this input power level (region $A$ in Figure 2). At increased $V_{\mathrm{ds}}$ and increased $\mathrm{V}_{\mathrm{gs}}$ the $\mathrm{RF}$ current exceeds that observed at DC conditions due to reduced or even vanishing self-heating in the dynamic case (region B). The negative output conductance in the dynamic case is determined by the slope of that trace along which the biasing has been performed (here, $\mathrm{V}_{\mathrm{gs}}=-2.5 \mathrm{~V}$ ). It should be noted that the additional RFpower is only a fractional amount of the DC-power (e.g. $15 \mathrm{~mW}$ vs $600 \mathrm{~mW}$ at $\mathrm{V}_{\mathrm{ds}}=16 \mathrm{~V}$ and $\mathrm{V}_{\mathrm{gs}}=-2.5 \mathrm{~V}$ ).

Reconstruction of the dynamic output characteristics has to be done carefully, since it depends on the power level: If at low $\mathrm{V}_{\mathrm{ds}}$ the $\mathrm{RF}$ input voltage amplitude is close to the cut-off boundary and biasing is performed step by step to higher $\mathrm{V}_{\mathrm{ds}}$ along a trace with large negative output conductance, the amplitude successively exceeds the cut-off boundary and therefore the resulting current will successively increase due to self-biasing (increasing asymmetrical waveform clipping with increasing exceed of the cut-off boundary).

Since heating leads to dispersion, large-signal modelling consequently requires rather data close to real operating conditions, i.e. the dynamic RF power data, than DC results $(5,6)$ for a proper description in simulation tools.

\section{MODELLING}

The large-signal model includes parasitic elements and the intrinsic transistor with three nonlinear current sources and two nonlinear capacitances. The gate current (gate-source and gate-drain current sources) is modelled by a usual Schockley diode equation, whereas for the drain current source the expression (1), which is divided into three parts separately accounting for the transfer behaviour $\left(f_{1}\right)$, output conductance $\left(f_{2}\right)$ as well as the triode region $\left(f_{3}\right)$, is used:

$$
\begin{gathered}
I_{d s}\left(V_{g s i}, V_{d s i}\right)=f_{1}\left(V_{g s i}\right) \cdot f_{2}\left(V_{g s i}, V_{d s i}\right) \cdot f_{3}\left(V_{d s i}\right) \\
f_{1}\left(V_{g s i}\right)=C D_{v c} \cdot\left(1+\tanh \left[\beta \cdot\left(V_{g s i}-V_{c}\right)+\gamma \cdot\left(V_{g s i}-V_{c}\right)^{3}\right]\right)+C D_{v s b} \cdot\left(1+\tanh \left[\delta \cdot\left(V_{g s i}-V_{s b}\right)\right]\right) \\
f_{2}\left(V_{g s i}, V_{d s i}\right)=1+\frac{\lambda}{1+\Delta_{\lambda} \cdot\left(V_{g s i}-V_{t o}\right)^{2}} \cdot V_{d s i}, \quad \text { with } \quad V_{t o}=V_{c}-\frac{2}{\beta} \\
f_{3}\left(V_{d s i}\right)=\tanh \left(\alpha \cdot V_{d s i}\right)
\end{gathered}
$$

The description of the equivalent circuit and these basic analytical equations may be found in refs. $(6,7)$. For the model parameter extraction the already presented RF output characteristics was used. Small-signal Sparameter measurements have been performed first (i) to extract the parasitic elements as well as (ii) to determine the gate voltage dependence of the non-linear gate capacitance at $V_{d s}=0 \mathrm{~V}$ and (iii) to find gate charge distribution at different drain voltages for analytical fitting of the parameters. Better agreement between simulated and measured S-parameters was achieved using the RF output characteristics instead of DC data for the model parameter extraction. In the RF case the negative character of the output conductance is not so pronounced, since self-heating is a low frequency phenomenon and cannot be observed during Sparameter measurements. As an example, we show in Figure 3 the resulting output characteristics, which reveal satisfactory agreement despite the fact that thermal effects have not yet been taken into account. 


\section{CONCLUSION}

The combination of power measurement results obtained by the use of a microwave transition analyser with an analytical large-signal model was demonstrated for nitride-based HEMTs. For these devices showing dispersion effects, this combination appears in particular convincingly. The large-signal model used has to be improved by implementing thermal effects to account for the negative output conductance. Further work concentrates also on simulations such as gain or output power of fundamental and higher harmonics, whereby the latter are also experimentally available by use of the microwave transition analyser.

\section{ACKNOWLEDGEMENT}

Financial support by Forschungsschwerpunkt Baden-Württemberg is gratefully acknowledged.

\section{REFERENCES}

(1) O. Ambacher, J. Smart, J.R. Shealy, N.G. Weimann, K. Chu, M. Murphy, W.J. Schaff, L.F. Eastman, R. Dimitrov, L. Wittmer, M. Stutzmann, W. Rieger, and J. Hilsenbeck; Two-dimensional Electron Gases Induced by Spontaneous and Piezoelectric Polarisation Charges in $\mathrm{N}$ - and Ga-Face AlGaN/GaN Heterostructures; 1999; J. Appl. Phys. 85, 3222-3233.

(2) E. Kohn, I. Daumiller, P. Schmid, N.X. Nguyen, and C.N. Nguyen; Large Signal Frequency Dispersion of AlGaN/GaN Heterostructure Field Effect Transistors; 1999; Electr. Lett. 35, 1022-1024.

(3) B.M. Green, K.K. Chu, M. Chumbes, J.A. Smart, J.R. Shealy, and L. Eastman; The Effect of Surface Passivation on the Microwave Characteristics of Undoped AlGaN/GaN HEMT's; 2000; IEEE Electr. Device Lett. 21, 268-270.

(4) B. Green, H. Kim, V. Tilak, J.R. Shealy, J.A. Smart, L.F. Eastman; Validation of an Analytical Large Signal Model for AlGaN/GaN HEMT's on SiC Substrates; 2000; Seventeenth Biennal IEEE/Cornell University Conference on Advanced Concepts in High Performance Devices.

(5) P.J. Tasker, M. Demmler, M. Schlechtweg, and M. Fernandez Barciela; Novel Approach to the Extraction of Transistor Parameters from Large Signal Measurements; 1994; $24^{\text {th }}$ European Microwave Conference, pp. 1301-1306.

(6) M. Demmler, P.J. Tasker, J.G. Leckey, and M. Schlechtweg; The Determination of the Transistor Dynamic I-V Characteristics from Large Signal RF Measurements; 1995; 25 ${ }^{\text {th }}$ European Microwave Conference, pp. 553-557, and references therein.

(7) M. Berroth, "Schaltkreissimulator für Heterostrukturfeldeffekttransistoren", Dissertation RuhrUniversität Bochum (1991).

(8) O. Breitschädel, H. Gräbeldinger, B. Kuhn, F. Scholz, W. Walthes, M. Berroth, I. Daumiller, K.B. Schad, E. Kohn, H. Schweizer; Short-channel AlGaN/GaN HEMTs with $70 \mathrm{~nm}$ T-gate; 1999; Electronic Letters 35, 2018-2019.

(9) M. Demmler, "Vektorkorrigiertes Großsignal-Meßsystem zur nichtlinearen Charakterisierung von Mikrowellentransistoren”, Dissertation Universität Karlsruhe (1996). 


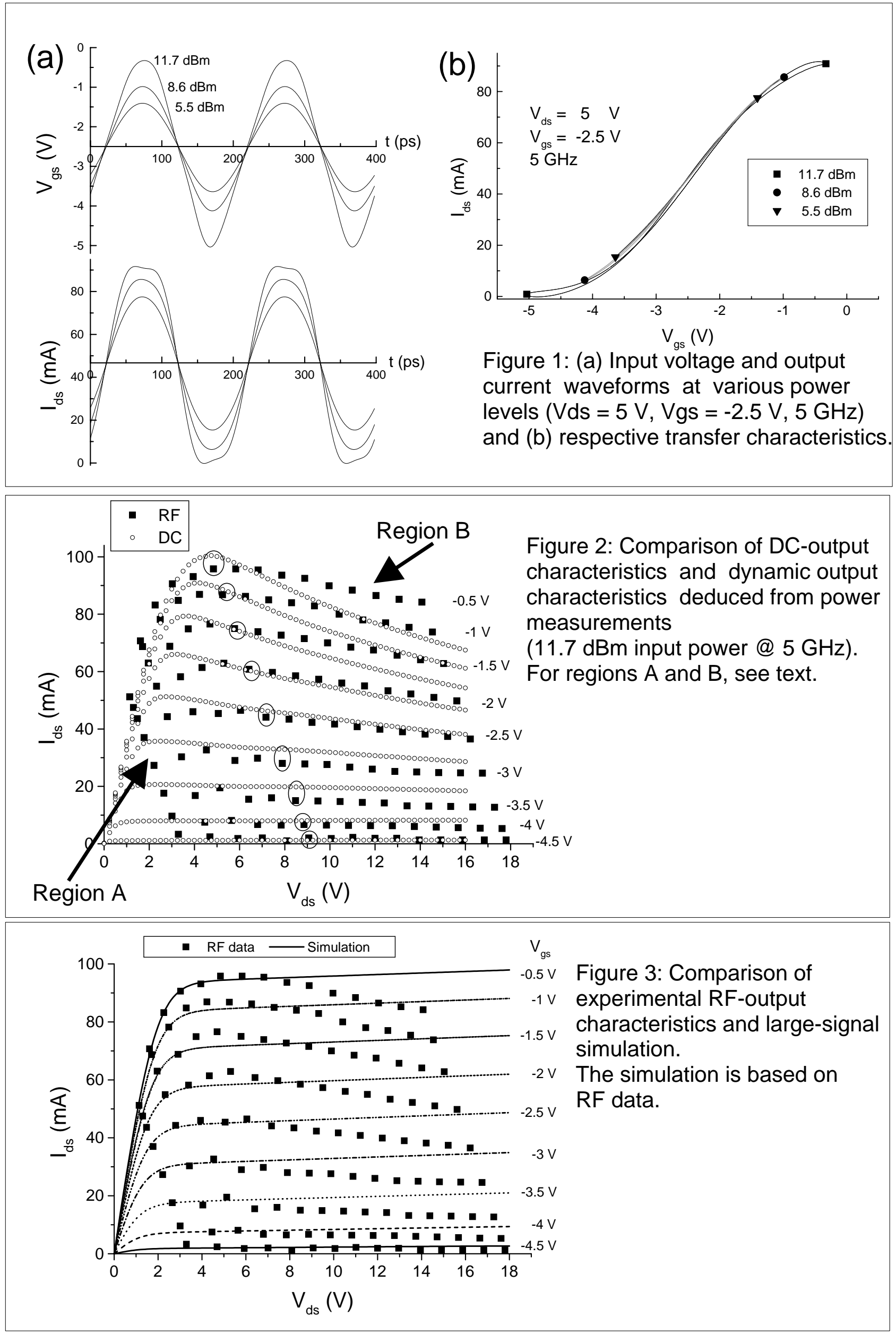

